\title{
Science et littérature : mélange des genres
}

Genèse de l'exposition « Science/Fiction, voyage au cœur du vivant »

\section{Claire Lissalde}

\section{(2) OpenEdition \\ Journals}

Édition électronique

URL : http://journals.openedition.org/aes/888

DOI : $10.4000 /$ aes.888

ISSN : 2258-093X

Éditeur

Laboratoire LISAA

Référence électronique

Claire Lissalde, "Science et littérature : mélange des genres », Arts et Savoirs [En ligne], 7 | 2016, mis en ligne le 07 décembre 2016, consulté le 10 décembre 2020. URL : http://journals.openedition.org/ aes/888; DOI : https://doi.org/10.4000/aes.888

Ce document a été généré automatiquement le 10 décembre 2020.

Centre de recherche LISAA (Littératures SAvoirs et Arts) 


\title{
Science et littérature : mélange des genres
}

Genèse de l'exposition « Science/Fiction, voyage au cœur du vivant »

\author{
Claire Lissalde
}

1 La médiation scientifique pose la question du médium, de l'interface que l'on veut construire entre la science et ses publics. Nous avons mené, au sein du pôle audiovisuel de l'Inserm (Institut national de la santé et de la recherche médicale), une réflexion sur les contours de cette médiation scientifique, en particulier sur le rôle de l'image, de la manière dont on peut raconter la science par l'image. Un aboutissement de notre réflexion a été la réalisation de l'exposition Science/Fiction, voyage au cœur du vivant ${ }^{1}$.

\section{Genèse d'un projet}

2 En 2007, l'Inserm choisit de développer une nouvelle politique de communication en allant à la rencontre du grand public. Cela se traduit par le lancement d'un site internet désormais plus informatif qu'institutionnel, la production de courts métrages diffusés sur le web et de longs-métrages pour la télévision et la réalisation d'expositions.

Les images de l'Inserm sont jusqu'à présent rassemblées dans une base de données, la photothèque Serimedis, qui compte 15000 photographies et 1500 vidéos. $C$ base fournit à la presse, à l'édition, à l'enseignement et à la recherche des photos et des séquences issues de nos laboratoires. En conformité avec la nouvelle politique de communication de l'Inserm, nous avons souhaité explorer de nouvelles vocations pour ces images. Nous consacrons ainsi beaucoup de temps à enrichir cette photothèque, à la faire vivre autrement que comme stock "inerte» d'images accumulées. Nous cherchons à la «rentabiliser", à mobiliser au maximum ses potentialités, à inventer des usages détournés comme autant de petits objets multimédias.

Les images scientifiques sont souvent belles, parfois magnifiques, mais elles sont difficilement accessibles. Elles dégagent une esthétique puissante et imprévue - imprévue car leur finalité première n'est pas de séduire, mais d'expliquer. Ce que ces images 
montrent n'est pas du tout familier du public, ce sont des pans du vivant normal ou pathologique invisibles à l'œil nu. Par ailleurs, ces images sont souvent utilisées à seul but d'illustration, pour «égayer " un texte. Mais nous souhaitons faire plus qu'illustrer ou décorer : nous voulons informer, communiquer, expliquer. L'image scientifique seule ne le peut pas : comme telle, elle ne raconte pas d'histoire à celui qui la contemple.

\section{De la base de données au récit}

La recherche d'une médiation scientifique nous amène ainsi à passer de la base de données au récit, selon les termes de la forte réflexion sur la création contemporaine posée par Lev Manovich ${ }^{2}$. La base de données est la figure organisatrice de notre époque numérique. Elle a une dimension purement horizontale de stockage d'informations mises à disposition, sans hiérarchie ni finalité. La construction de récit commence quand on opère une sélection dans cette base de données. On sépare, on trie, on choisit, on copiecolle... on passe de la donnée à l'ordonné, à l'objet narratif conçu en vue d'une certaine interaction avec son public.

6 La science est, elle aussi, une narration mais comme pratique professionnelle, elle se destine à n'être lue et interprétée que par les spécialistes capables de la comprendre et de la construire. Dans la médiation scientifique, le public n'est pas composé de chercheurs ni d'ingénieurs en mesure de comprendre le discours interne de la science. La science ellemême est devenue une activité tellement spécialisée que l'image issue d'un laboratoire n'est pas forcément comprise par le laboratoire voisin... alors que dire du grand public?

Une image remplace mille mots, dit-on souvent. Ce n'est pas exact dans le cas de l'image scientifique. Utilisée autrement que comme preuve dans une publication de recherche de rang $\mathrm{A}$, cette image a absolument besoin d'être accompagnée d'une explication. Nous avons choisi pour la rendre abordable une hybridation: mélange de genres, mélange de formats et mélange de temps.

\section{Science et littérature, de Jules Verne à Bernard Werber}

Construire un récit, mais à partir d'où ? Mélanger science et fiction, mais sur quelle base ?

Afin de tester des modes de narration, j'ai un jour demandé à Éric Dehausse (iconographe au pôle audiovisuel) de me raconter une histoire, celle d'une cellule à qui il arriverait de multiples aventures (rencontres, ruptures, maladies, etc.). Dans la discussion, Éric fait allusion à ses moyens mnémotechniques : il a l'habitude de mémoriser certaines images très demandées en les rapportant à des illustrations de Jules Verne, auteur dont il a conservé depuis l'enfance les souvenirs des magnifiques illustrations de ses œuvres. Illustrations et non textes : c'est bien ici l'image qui renvoie au souvenir.

Aussi, le choix du géant français de la littérature d'anticipation s'impose de lui-même: Jules Verne a très largement participé à la vulgarisation des découvertes scientifiques du $\mathrm{XIX}^{\mathrm{e}}$ siècle. Il s'inscrit dans un imaginaire collectif vivant, il est un pan de la culture populaire. À son époque, son éditeur Pierre-Jules Hetzel publie déjà la Bibliothèque illustrée des Familles, puis Le Magasin d'éducation et de récréation: il est persuadé que l'image vient à l'appui de la connaissance. Jules Verne, d'abord réticent, finit par se laisser convaincre. Le succès des ventes est immense et l'image $\mathrm{y}$ contribue grandement. Le succès 
intergénérationnel des Voyages extraordinaires a montré la fécondité de cette intuition. Près de 200 ans après sa parution, cette série de romans et nouvelles reste une référence.

11 Nous aussi, à la manière de Hetzel, nous avons en quelque sorte décidé de mieux «vendre» la science, d'attirer vers elle sans effrayer, en partant d'images connues et populaires, « déjà vues ». Le projet d'exposition prend forme, nous lui donnons son nom : Science/Fiction, voyage au coeur du vivant. Le principe: partir de l'image scientifique, la détourner et la mixer à une illustration ancienne, lui adjoindre des récits fictionnels et pédagogiques. Nous avons alors cherché une plume contemporaine et identifié l'écrivain populaire Bernard Werber ${ }^{3}$, dont l'œuvre a un immense succès en France et dans le monde. Il se trouve que Jules Verne est aussi l'écrivain favori de Bernard Werber. Celui-ci accepte de nous accompagner et il choisit le principe d'une écriture automatique de courts récits fictionnels face aux tableaux de l'exposition. Lors de son écriture, il ne souhaite pas connaître les légendes scientifiques des images, la seule contrainte que nous avons posée à son imagination est de ne pas user de connotations médicales ou biologiques qui pourraient induire le spectateur en erreur. Il explique :

Dans le titre Science/Fiction, voyage au cœur du vivant, il y a: Science, c'est l'endroit où les choses bougent, où cela va faire changer le monde; Fiction: on n'est pas bloqué par le réel, on peut aller au-delà de la science. Il faut surprendre car la science est aussi un divertissement, un spectacle. ${ }^{4}$

12 Les récits microfictionnels de Bernard Werber ont été réalisés en mode d'écriture automatique et intuitive face à l'image, l'exercice faisant l'objet d'une vidéo.

\section{Choix de sens, choix du format}

13 Le matériau de base de l'exposition est formé par des photographies scientifiques venant des laboratoires de recherche de l'Inserm. Elles sont issues d'observations réalisées au microscope, par scanner, par échographie ou tout autre moyen d'imagerie.

Par le choix des images, nous avons couvert les grands champs de recherche de l'Inserm : neurosciences, cancer, microbiologie et maladies infectieuses, immunologie, génétique etc... Nous avons demandé à un journaliste scientifique, Charles Muller, de «traduire » les légendes scientifiques originales des photographies dans un langage vulgarisé et accessible au plus grand nombre. Ces images se mêlent à l'univers de Jules Vernes grâce aux montages talentueux d'Éric Dehausse d'une part et, d'autre part, aux courtes narrations décalées de Bernard Werber. Elles amusent, étonnent; les petits textes explicatifs quant à eux, instruisent. Différents niveaux de récits se croisent ainsi autour de l'image, formant autant de portes d'accès pour le spectateur.

Les photomontages sont réunis dans 28 tableaux. Nous avons scénographié ces photomontages, en restant dans cet univers jules-vernien, dans un style industriel «Eiffel » de la fin du XIX ${ }^{e}$ siècle. Nous avons enrichi la scénographie d'un grimoire géant posé au sol: le visiteur peut feuilleter ce livre en recomposant lui-même les surimpressions d'images. Les pages s'en échappent, investissant l'espace d'exposition où le public peut se promener le temps d'un voyage ludique et pédagogique.

Nous avons demandé aux scientifiques, auteurs des photos, d'autoriser le "détournement» graphique de leur œuvre originale et tous ont accepté avec enthousiasme. C'est aussi le cas pour le Musée Jules-Verne de la ville de Nantes, dont la directrice Agnès Marcetteau nous a donné son accord. Agnès Marcetteau a montré un 
enthousiasme et un soutien constants pour le projet. Elle a mis à notre disposition les versions haute définition des gravures originales illustrant les œuvres de Jules Verne. Nous avons ainsi joué des genres, mais dans ce détournement, nous avons aussi tenu à respecter les œuvres originales et leurs auteurs, à ne pas les trahir. Ce détournement a été maintes fois qualifié d'«audacieux», parfois même de "culotté», mais jamais de " déplacé».

\section{Fictionnaliser la science}

Nous avons signalé le pouvoir évocateur, fascinant même des images scientifiques produites par les technologies de microscopie. Cette fascination est aussi un piège : en rester au caractère mystérieux des formes dont on admire la beauté mais dont on ne comprend pas le sens.

18 L'attrait de l'image scientifique, doublée ici du collage avec la gravure de l'œuvre originale de Jules Verne est exploité comme invitation à la connaissance. Le spectateur n'est pas limité à un plaisir esthétique de contemplation, de fascination, mais plutôt convié à entrer dans la connaissance. Chaque tableau est un portique vers un " pour en savoir plus ». C'est un circuit initiateur, il doit donner faim de science, ouvrir l'appétit de connaissance.

19 La scénographie de l'exposition Science/Fiction, voyage au cœur du vivant rejoint ainsi les intuitions exprimées par Hugo Gernsback, fondateur des Amazing Stories (1926): " la science-fiction ne doit pas seulement divertir, elle doit aussi "éduquer", c'est-à-dire inciter ses lecteurs à découvrir, par le détour fictionnel, les potentialités civilisatrices des sciences et des technologies " ${ }^{5}$. On sait que Gernsback a fait le choix réussi à l'époque d'un graphisme choc, aux couleurs vives et aux typographies frappantes caractéristiques du style Pulps, pour engager le lecteur à découvrir des «romances scientifiques». Il a importé Jules Verne aux États-Unis et créé le label « science-fiction », ouvrant une veine féconde jusqu’à nos jours.

\section{De l'exposition au Musée virtuel}

L'exposition a très bien circulé : présentée dans 28 pays, elle a été traduite en anglais, en espagnol, en portugais et en arabe. Elle a été portée par la double notoriété internationale de Jules Verne et de Bernard Werber. Dès son lancement, en décembre 2009, nous avons fait le choix de diversifier les lieux, c'est-à-dire de poursuivre le "mélange des genres » dans l'accueil des publics : Cloître du Groupe hospitalier Cochin, Festival «Utopiales » de Nantes, Médiathèque de la Cité des sciences et de l'industrie, bibliothèques municipales... jusqu'aux centres commerciaux. L'image d'exposition peut sortir des lieux habituellement consacrés pour investir des territoires nouveaux, plus proche des gens.

21 L'expérience nous montre cependant qu'il existe une réticence du réseau culturel à accueillir des expositions traitant de science, quand bien même l'approche choisie serait détournée, ludique et pédagogique; la diffusion de la science dans la société souffre encore de certains préjugés sur sa complexité et son élitisme présumés.

Nous avons alors choisi Internet pour ouvrir encore plus l'accès à la science racontée, en concevant un lieu ouvert $24 \mathrm{~h} / 24 \mathrm{~h}$ et $7 \mathrm{j} / \mathrm{j}$ : Le Musée virtuel. Situé dans un vaisseau spatial modélisé en 3D, le Musée virtuel poursuit l'existence des expositions de l'Inserm à travers un parcours immersif et interactif, largement connecté aux réseaux sociaux. 
es salles numériques hébergeant l'exposition Science/Fiction, voyage au cœur du vivant sont conçues dans une scénographie Steam Punk, uchronique en mode "futur antérieur », en poursuivant le mélange des genres par un mélange de temps, puisque de nombreux inserts de documents nous raccordent directement dans le présent (et l'avenir) du xxI siècle. La société SISSO, dirigée par des jeunes férus de science-fiction et déjà ancrés dans le milieu culturel, a montré une grande élégance dans le choix scénographique et esthétique des salles du musée. La réalisation est multimédia et poursuit le mélange des genres par le mélange des supports...

Autre idée dérivée de l'exposition : nous avons proposé à François Demerliac, réalisateur, de raconter des histoires de science en embarquant le capitaine Nemo dans le corps humain, en s'inspirant du film de Richard Fleisher Le voyage fantastique (1966). 20 épisodes ont été ainsi coproduits et transportent désormais le savoir "à bord du Nanotilus ${ }^{6}$, navire imaginaire circulant dans les réseaux numériques pour atteindre le cerveau des jeunes spectateurs curieux de découvertes.

\section{La science à l'ère pop}

La culture populaire offre un imaginaire collectif et un récit partagé par les générations. Elle est une porte d'entrée vers d'autres univers, dont celui de la science, qui n'a pas à craindre de cette proximité. On peut aujourd'hui « venir pour l'art et repartir avec la science ", observait le physicien et grand vulgarisateur Brian Greene. L'exposition Science/Fiction, voyage au cour du vivant a été pour nous une expérimentation : copier-coller, hybrider, détourner, assembler, mixer et remixer la pop culture et la science d'avant-garde.

\section{[[vueexpo]]}

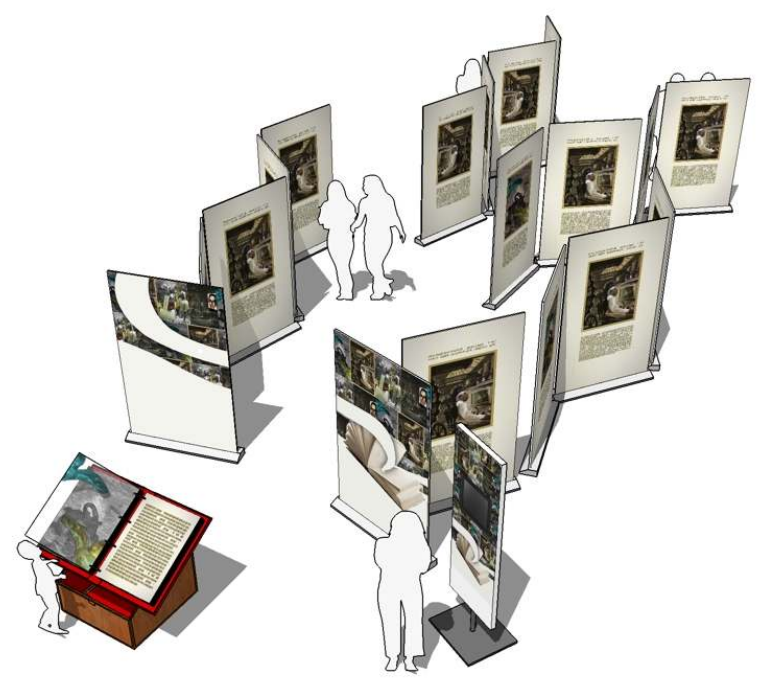

Éléments scénographiés, grimoire (au premier plan à gauche) et tableaux. (C)Inserm/Ogilvy 
[[0139]]

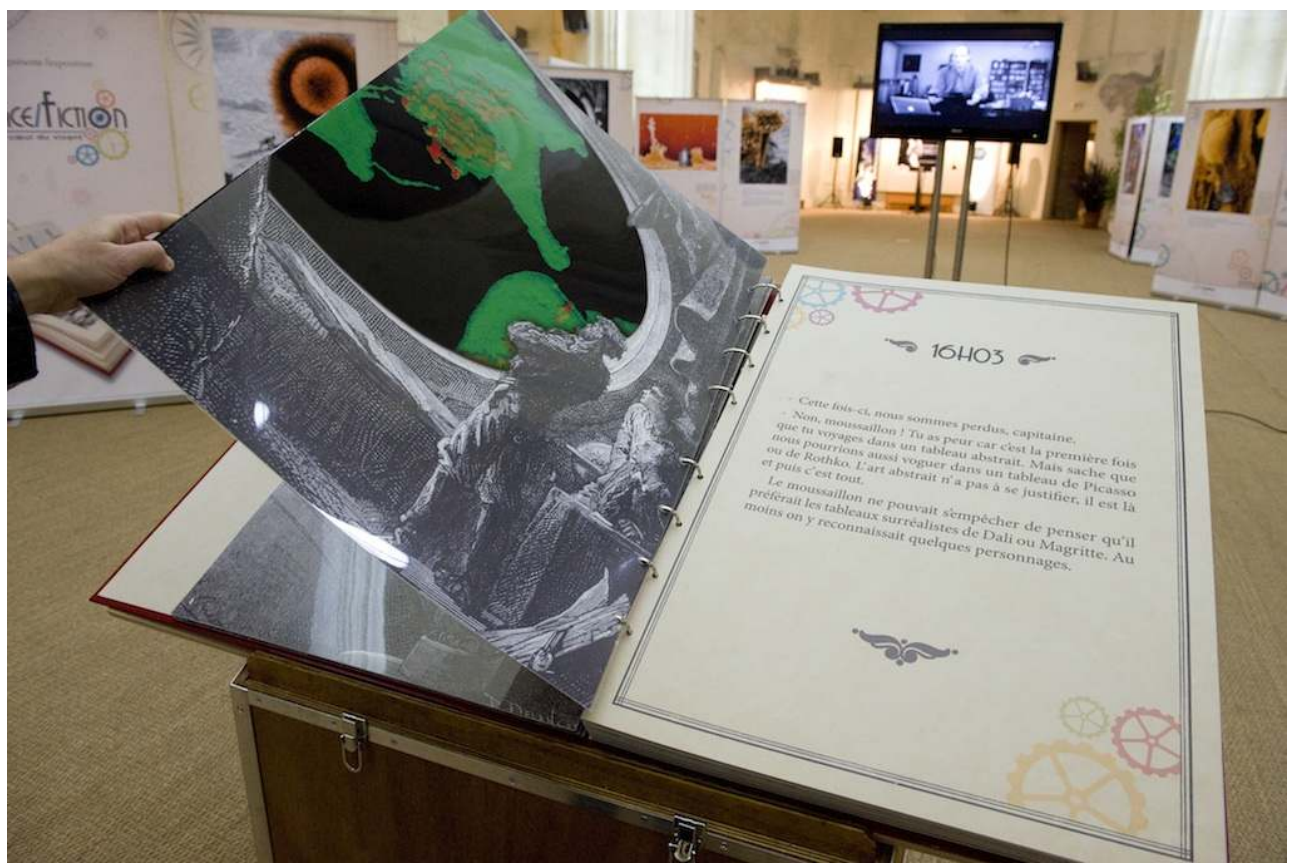

Le grimoire dans le contexte de l'exposition. Décembre 2009, Espace Saint-Rémi, à Bordeaux (C)Inserm-Ogilvy/E. Begouen 


\section{[[inserm_50623]]}

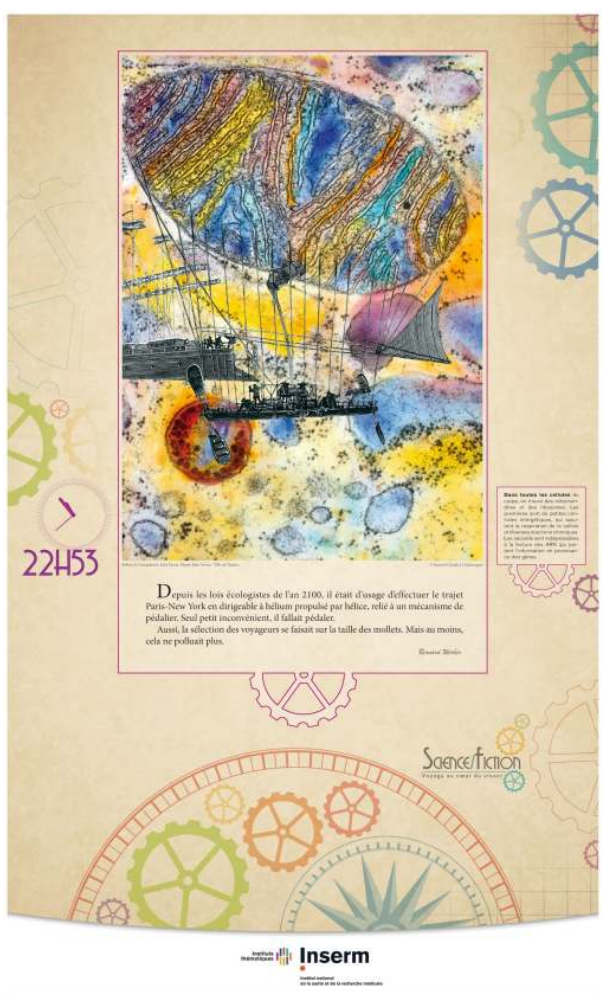

Un tableau de l'exposition qui allie l'énergie cellulaire à l'énergie « écolo ».

Texte de Bernard Werber : « Depuis les lois écologistes de l'an 2100, il était d'usage d'effectuer le trajet Paris-New York en dirigeable à hélium propulsé par hélice, relié à un mécanisme de pédalier. Seul petit inconvénient, il fallait pédaler. Aussi, la sélection des voyageurs se faisait sur la taille des mollets. Mais au moins, cela ne polluait plus. »

Texte de la légende pédagogique : «Dans toutes les cellules du corps, on trouve des mitochondries et des ribosomes. Les premières sont de petites centrales énergétiques, qui assurent la respiration de la cellule et diverses réactions chimiques. Les seconds sont indispensables à la lecture des ARN qui portent l'information en provenance des gènes. »

Photo scientifique, coloration manuelle de Claude Le Goascogne

(c) Inserm C. Le Goascogne-Robur le conquérant. Jules Verne - Musée Jules Verne - Ville de Nantes. Photomontage E. Dehausse 
(Inserm 50653)

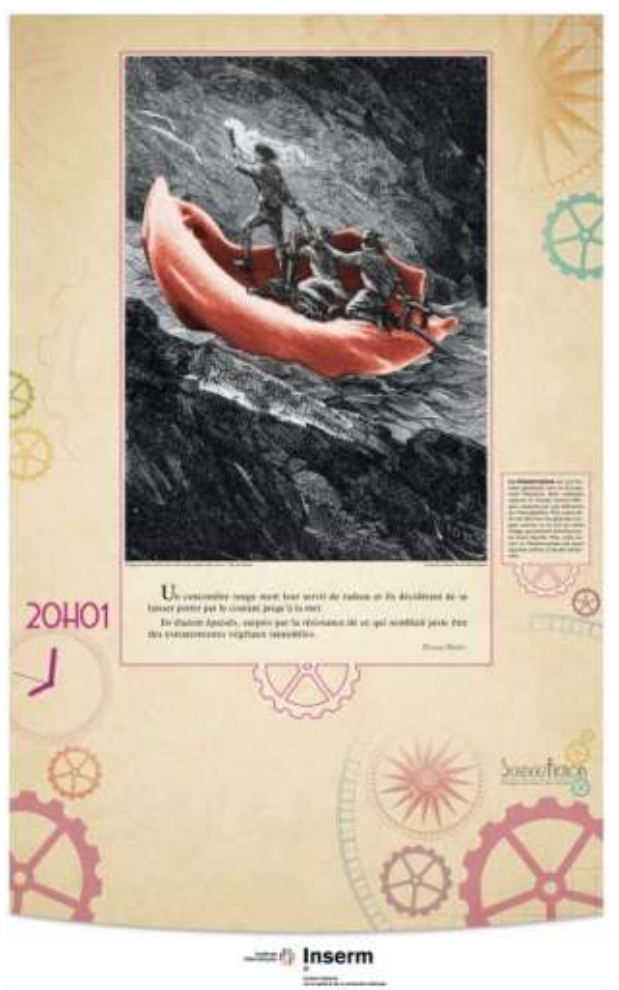

Un concombre rouge mort leur servit de radeau et ils décidèrent de se laisser porter par le courant jusqu'à la mer.

Ils étaient épuisés, surpris par la résistance de ce qui semblait juste être des extraterrestres végétaux immobiles.

La drépanocytose est une maladie génétique rare en Europe, mais fréquente dans certaines régions du monde comme l'Afrique, marquée par une altération de l'hémoglobine. Elle a pour effet de déformer les globules rouges, comme on le voit sur cette image, qui prennent la forme courbe d'une faucille. Pour cette raison, la drépanocytose est aussi appelée anémie à cellules falciformes.

(c) Inserm/Claude Féo \& Marcel Bessis, Voyage au centre de la Terre, Jules Verne. Musée Jules VerneVille de Nantes

\section{[[SF_musee]]}

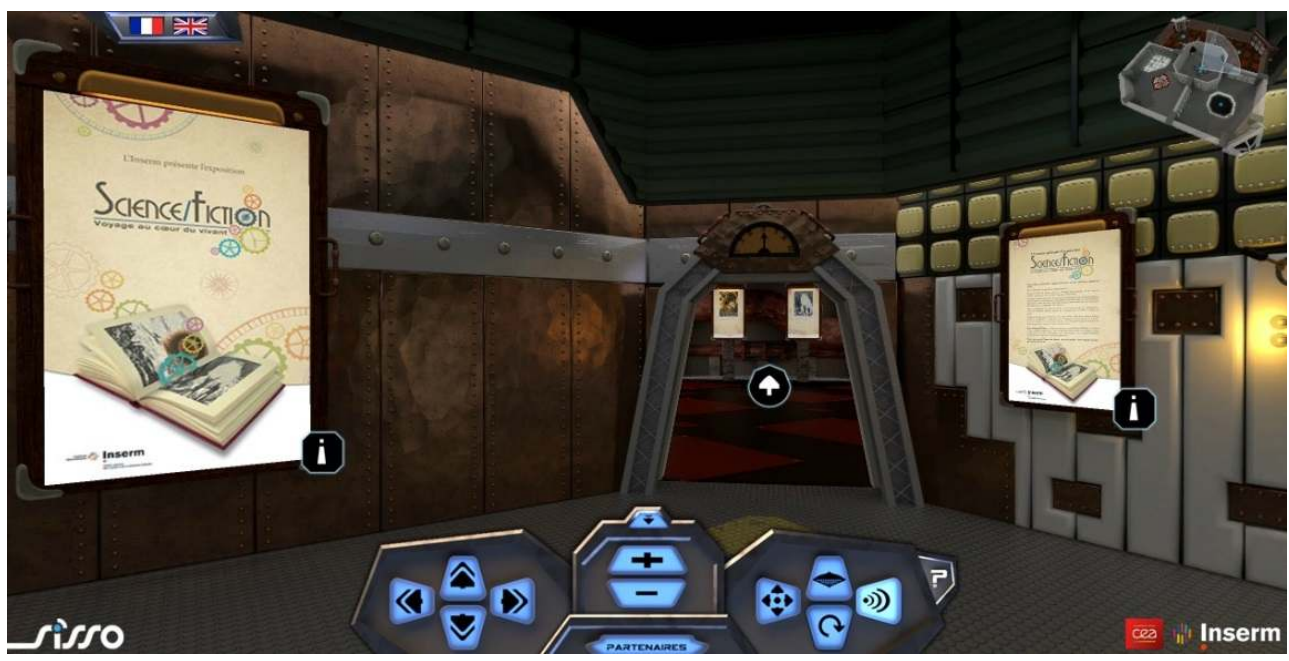

Entrée de l'exposition dans le Musée virtuel (musee.inserm.fr) (c)Inserm/Sisso. 
[[SF_musee2]]

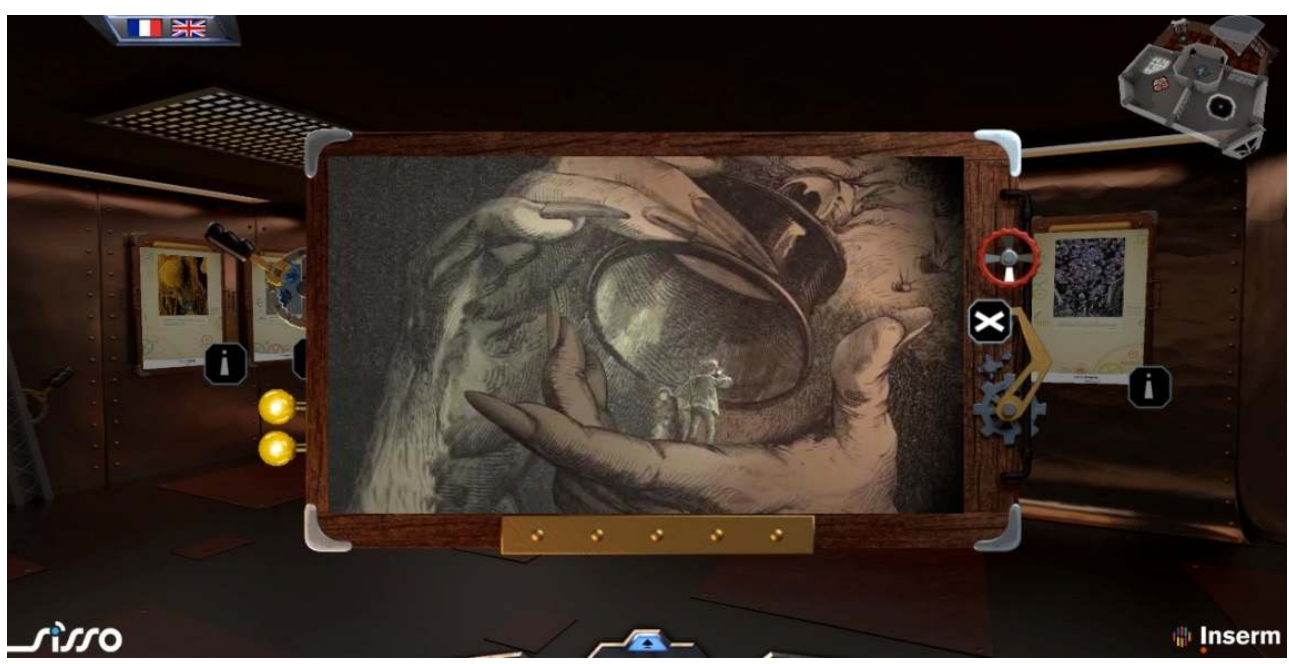

Diffusion des épisodes « À bord du Nanotilus » dans la salle modélisée du Musée virtuel (C)Inserm/Sisso.

(Inserm 46887)

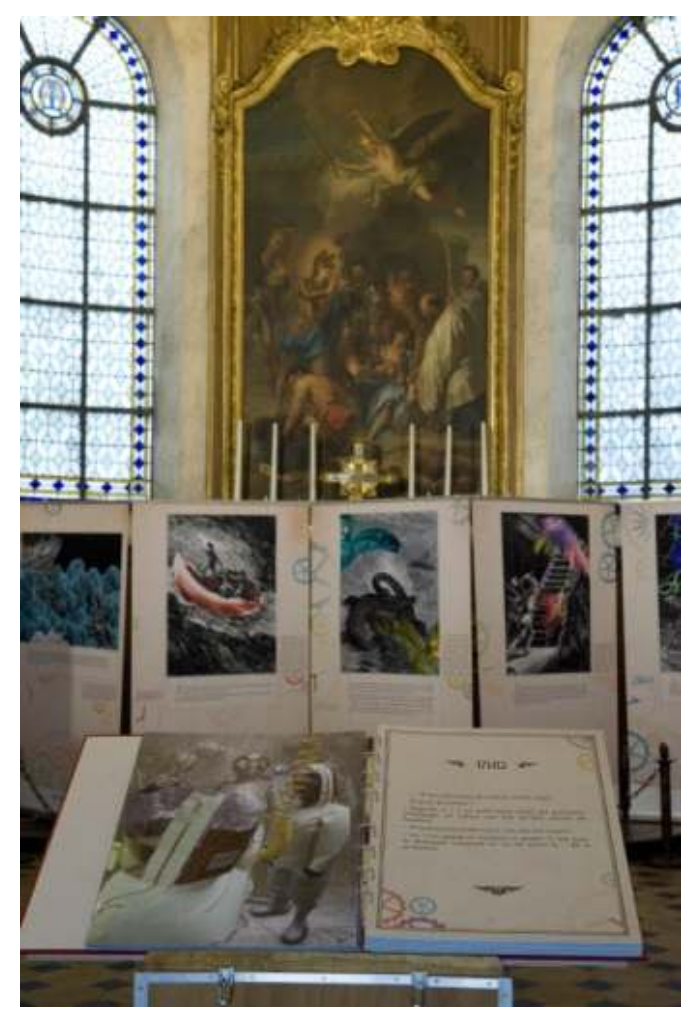

La chapelle des Gobelins, Paris 2010

COInserm/E. Begouen 
(Inserm 47094)

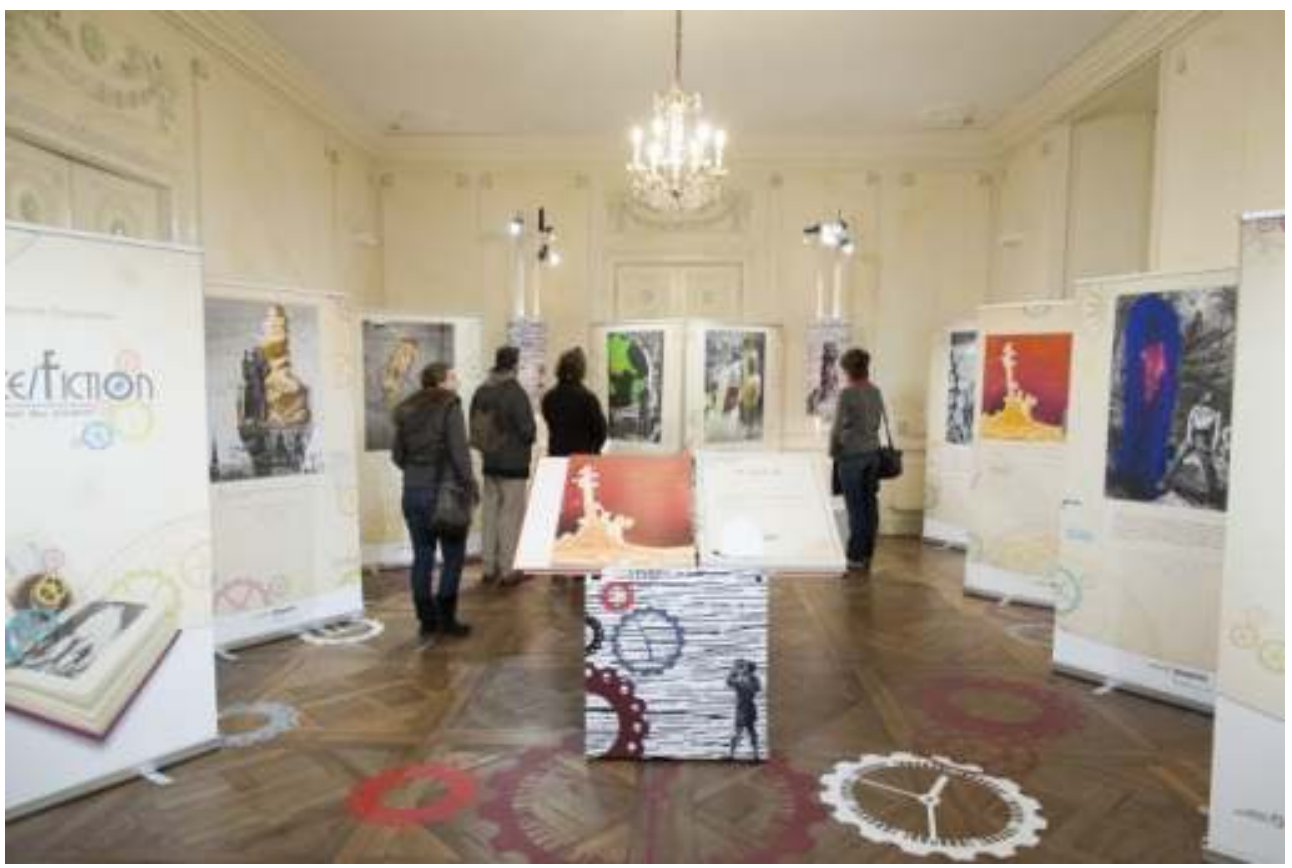

À Vannes, Hôtel de Limur, décembre 2013.

(C) F. Le Divenah

(Inserm 47706)

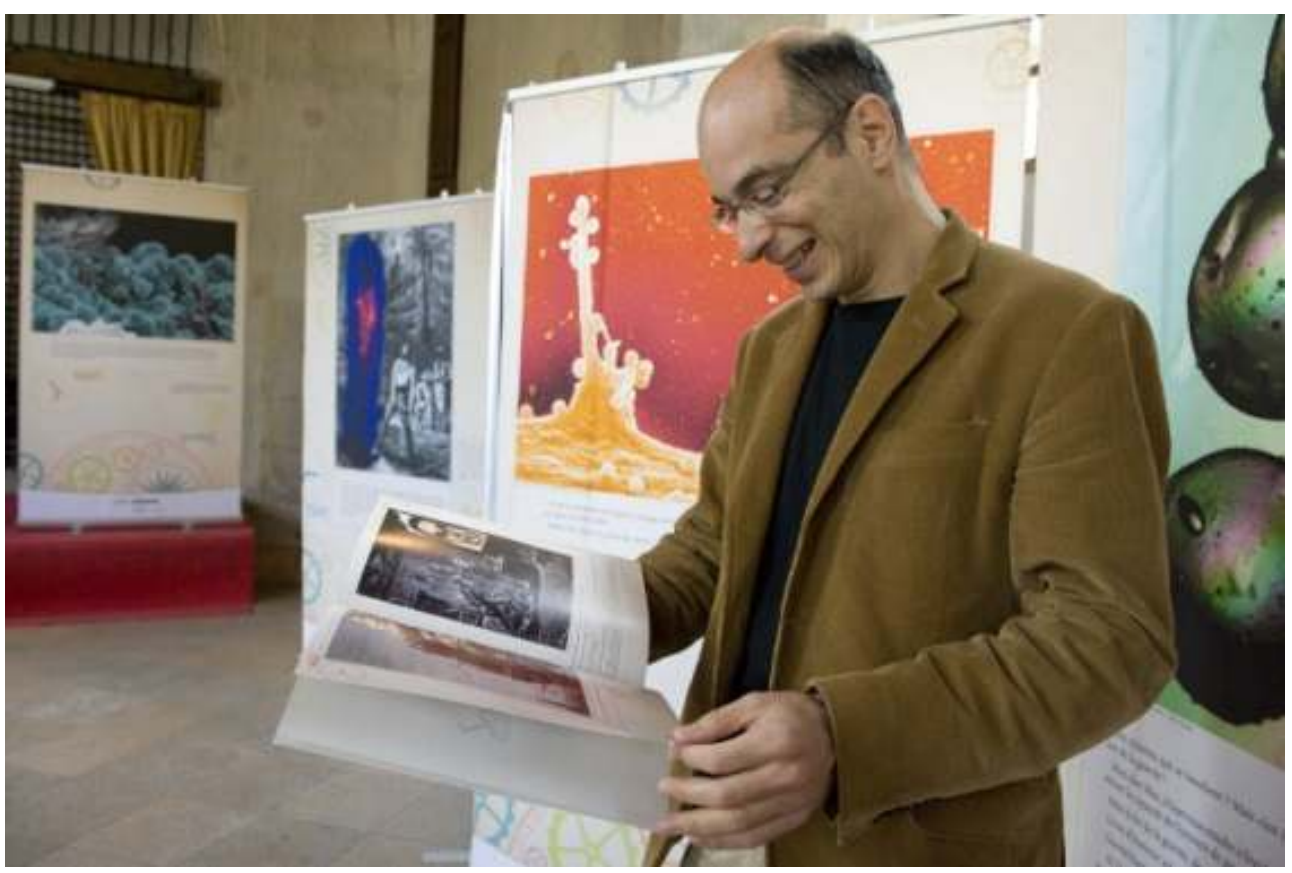

BERNARD WERBER lors de l'inauguration de l'exposition au Cloître du Groupe hospitalier Cochin - SaintVincent-de-Paul, salle du Cœur des Religieuses. Mai 2010

(C)Inserm/E.Begouen 


\section{NOTES}

1. Cette exposition, inaugurée en décembre 2009 à l'occasion du festival Cinémascience à Bordeaux, a été, en 2010, présentée aux Utopiales, festival international de Science fiction, à Nantes puis exposée à Paris à l'Assemblée nationale et au Couvent du centre de recherche des Cordeliers. En 2011, elle a été exposée à la Cité des Sciences et de l'industrie à Paris, au forum départemental des sciences à Lille, au Futuroscope de Poitiers, etc. Enfin, elle a voyagé dans plus de 30 pays en en Europe, Afrique, Asie et Amérique latine et a été traduite en anglais, espagnol, portugais, arabe.

2. Manovich, Lev, 2010, Le langage des nouveaux médias. Dijon, Les Presses du Réel.

3. Auteur de La Trilogie des fourmis (traduit dans 35 langues) et de très nombreux ouvrages dont Le souffle des dieux, Le rire du Cyclope etc.

4. Extrait de la vidéo Science fiction, voyage au cœur du vivant, Bernard Werber raconte. Production Inserm/AM productions. Durée : 7 min. Disponible au catalogue de films de l'Inserm : en ligne sur www.serimedis.inserm.fr (ref. 49785).

5. Cité par Marc Attalah, directeur de la Maison d'ailleurs (Musée des utopies), 2012.

6. http://serimedis.inserm.fr//zooms/00000000013/56111.h264

\section{RÉSUMÉS}

Cet article expose les démarches et les enjeux de l'organisation de l'exposition Science/Fiction, voyage au cour du vivant par l'INSERM. Il souligne également l'innovation proposée par une médiation qui utilise le mélange des genres littéraires et scientifiques pour atteindre son public.

\section{INDEX}

Mots-clés : médiation, exposition, science-fiction, Verne (Jules), Werber (Bernard)

\section{AUTEUR}

\section{CLAIRE LISSALDE}

Directrice du pôle audiovisuel de l'INSERM, Commissaire de l'exposition Science-Fiction, voyage au cour du vivant 